\title{
Antimicrobial and cytotoxic activities of 1,2,3-triazole-sucrose derivatives
}

\author{
Krasimira T. Petrova a,*, Taterao M. Potewar ${ }^{a}$, Paula Correia-da-Silva ${ }^{\text {b }}$, M. Teresa Barros a , \\ Ricardo C. Calhelha ${ }^{c}$, Ana Ćiric ${ }^{d}$, Marina Soković ${ }^{d}$, Isabel C. F. R. Ferreira ${ }^{c}$ \\ a LAQV, REQUIMTE, Departamento de Química, Faculdade de Ciências e Tecnologia, Universidade Nova de Lisboa, 2829-516 Caparica, Portugal \\ ${ }^{\mathrm{b}}$ Instituto Superior de Ciências da Saúde Egas Moniz, 2829-511 Caparica, Portugal \\ c Mountain Research Centre (CIMO), ESA, Polytechnic Institute of Bragança, Campus de Santa Apolónia, 1172, 5301 -855 Bragança, Portugal \\ d Department of Plant Physiology, Institute for Biological Research, "Siniša Stanković", University of Belgrade, Bulevar Despota Stefana 142, 11000 Belgrade, \\ Serbia
}

\section{A R T I C L E I N F O}

\section{Article history:}

Received 4 July 2015

Received in revised form 3 September 2015

Accepted 4 September 2015

Available online 15 September 2015

\section{Keywords:}

1,2,3-Triazoles

Sucrose

Antibacterial activity

Antifungal activity

Cytotoxic activity

\begin{abstract}
A B S T R A C T
A library of $1-\left(1^{\prime}, 2,3,3^{\prime}, 4,4^{\prime}, 6\right.$-hepta-O-acetyl-6'-deoxy-sucros-6'-yl)-1,2,3-triazoles have been investigated for their antibacterial, antifungal and cytotoxic activities. Most of the target compounds showed good inhibitory activity against a variety of clinically and food contaminant important microbial pathogens. In particular, 1-(1',2,3,3',4,4',6-hepta-O-acetyl-6'-deoxy-sucros-6'-yl)-4-(4-pentylphenyl)-1,2,3triazole (5) was highly active against all the tested bacteria with minimal inhibitory concentrations (MICs) ranging between 1.1 and $4.4 \mu \mathrm{M}$ and bactericidal concentrations (MBCs) from 2.2 and $8.4 \mu \mathrm{M}$. The compound 1-(1',2,3,3',4,4',6-hepta-O-acetyl-6'-deoxy-sucros-6'-yl)-4-(4-bromophenyl)-1,2,3-triazole (3) showed antifungal activity with MICs from 0.6 to $4.8 \mu \mathrm{M}$ and minimal fungicidal concentrations (MFCs) ranging between 1.2 and $8.9 \mu \mathrm{M}$. Furthermore, some of the compounds possessed moderate cytotoxicity against human breast, lung, cervical and hepatocellular carcinoma cell lines, without showing toxicity for nontumor liver cells. The above mentioned derivatives represent promising leads for the development of new generation of sugar-triazole antifungal agents.
\end{abstract}

(c) 2015 Elsevier Ltd. All rights reserved.

\section{Introduction}

The alarming rates of emerging and reemerging microbial threats coupled with the growing antimicrobial resistance to current antibiotics are major concerns to the public health and scientific communities worldwide. ${ }^{1,2}$ These trends have emphasized the urgent need for designing and developing new classes of antimicrobial agents with different chemical structures and mechanism of action compared with traditional drugs, in order to improve their activities while retaining good bioavailability and safety profiles. ${ }^{3}$

1,2,3-Triazole derivatives are an important class of heterocyclic compounds with various potential applications, ${ }^{4}$ which have aroused growing attention in recent years with the introduction of 'click chemistry' for their easy and efficient synthesis. ${ }^{5}$ The triazole is an attractive bridge group, which could connect two pharmacophores to produce novel bifunctional molecules, ${ }^{6}$ while it is almost impossible to be hydrolyzed, oxidized or reduced. Though 1,2,3-triazole units are not present in natural products, they are

\footnotetext{
* Corresponding author. Departamento de Química, Faculdade de Ciências e Tecnologia, Universidade Nova de Lisboa, Quinta da Torre, 2829-516 Caparica, Portugal. Tel.: +351937013742; fax: +351212948550.

E-mail address: k.petrova@fct.unl.pt (K.T. Petrova).
}

remarkably stable to metabolic transformations and are present in many drugs such as tazobactam, cefatrizine, fluconazole, voriconazole, itraconazole and posaconazole. ${ }^{7}$ New triazoles with improved pharmacological and pharmacokinetic profiles are emerging rapidly. ${ }^{8}$

Various glycosides can be found in natural resources, mainly in the form of glycoconjugates, such as glycopeptides, glycolipids, and nucleic acids, where the saccharide moiety plays important role for their biological activity. ${ }^{9}$ Considering that sugar moieties with polyhydroxyl groups have been extensively employed in drug design with the view to improve water solubility and to increase the interaction between receptors and guests for molecular recognition, ${ }^{10,11}$ various novel monosaccharide-derived 1,2,3-triazoles were synthesized and their inhibiting activities for glycosidases, such as $\alpha$-glucosidase, isomaltase, amyloglucosidase and $\beta$-glucosidase, ${ }^{12}$ sweet almond $\beta$-glucosidase and yeast $\alpha$-glucosidase, ${ }^{13}$ or a-glucosidase (Saccharomyces cerevisiae), $\beta$-glucosidase (almonds), $\alpha$-galactosidase (green coffee beans), $\beta$-galactosidase (Aspergillus oryzae), $\alpha$-mannosidase (Canavalia ensiformis), $\beta$-mannosidase (snail acetone powder), and $\beta$-N-acetylglucosaminidase, ${ }^{14}$ were tested. Others were tested against fucosidases,${ }^{15}$ trans-sialidase, ${ }^{16}$ glycogen phosphorylase, ${ }^{17}$ etc. Members of the sugar-triazole conjugates family were investigated for other biological activities, such as receptor interactions, ${ }^{18}$ antitubercular activity, ${ }^{19}$ nucleoside mimetics, ${ }^{20}$ 
and antiviral agents. ${ }^{21}$ Many of them have shown strong antibacterial and antifungal activities, for example glycal-derived tetrahydrofuran 1,2,3-triazoles, ${ }^{22}$ pyranoid derivatives comprising both triazole and conjugated carbonyl system, ${ }^{23} \mathrm{D}$-glucose-derived benzyl and alkyl 1,2,3-triazoles, ${ }^{24} 6$-triazolyl 2,3,6-trideoxy sugars, ${ }^{25}$ and triazoles with substituted triazole-piperidine side chains. ${ }^{26}$

Antitumor agents for chemotherapy also attract much attention, since cancer is responsible for many lethal outcomes worldwide. ${ }^{27}$ Derivatives of $4 \beta$-(1,2,3-triazol-1-yl)podophyllotoxin have been obtained and their cytotoxicity toward human cancer cell lines HL-60, A-549, HeLa and HCT-8 were assessed, showing potent anticancer activity toward HL-60 and moderate cytotoxicities against the rest of the studied cell lines. ${ }^{28}$

Sucrose, being a biorenewable, biocompatible and biodegradable raw material with relatively low cost, ${ }^{29}$ is a promising starting material for the synthesis of new compounds with biological activity. ${ }^{30}$ Our research group has been focused on the applications of sucrose for the synthesis of new compounds with potential applications either industrial or in academia. In this sense, we have developed sucrose chemoselective derivatization methods, ${ }^{31,32}$ the synthesis of sucrose-based biodegradable polymers ${ }^{33-35}$ and nanoparticles. ${ }^{36}$ To the best of our knowledge, the only other example in the literature of the synthesis of sucrose triazoles was reported by Jarosz et al. for the construction of sucrose macrocycles with complexation properties. ${ }^{37,38}$

Based on these literature data and the features described previously, we have created a small library of 1,2,3-triazoles of sucrose ${ }^{39}$ to be screened for their biological activities. ${ }^{40}$ Their antimicrobial and antifungal activities were tested and compared with the ones of some commercial antibiotics. Cytotoxicity against a number of human tumor cell lines and non-tumor liver cell primary culture was studied as well.

\section{Results and discussion}

\subsection{Chemistry}

The library of 1,2,3-triazole-sucrose derivatives is presented in Fig. 1 and have been synthesized as previously described. ${ }^{39}$ Briefly, the series of 1 - $\left(1^{\prime}, 2,3,3^{\prime}, 4,4^{\prime}, 6\right.$-hepta-O-acetyl-6'-deoxy-sucros-6'yl)-1,2,3-triazoles were obtained by microwave assisted copper catalyzed 1,3-dipolar cycloaddition of sucrose derived azides with terminal alkynes in excellent yields and in short reaction times. The compound $1^{\prime}, 2,3,3^{\prime}, 4,4^{\prime}, 6$-hepta-O-acetyl-6'-azido-6'-deoxysucrose was regioselectively synthesized from sucrose by improved procedure and used for the cycloadditions.

The antimicrobial and cytotoxic activities of the synthesized library of compounds have been studied in their peracetylated form. There are indications in the literature that hydrophobic groups as acetyls increase the molecule's tendency to aggregate on the cell membrane and facilitate its permeability. On the other hand, the presence of the acetyl groups can influence the enzymatic activity, triggering higher or lower affinity of the compound toward various enzymes involved in the processes. ${ }^{41}$

\subsection{Antibacterial activity}

The results of the antibacterial activity, evaluated by the microdilution method, of the 1,2,3-triazole-sucrose derivatives and standard antibiotics are presented in Table 1 . All derivatives showed antibacterial activity against all the tested bacteria with minimal inhibitory concentrations (MICs) ranging between 1.1 and $38 \mu \mathrm{M}$ and bactericidal concentrations (MBCs) from 2.2 and $57.2 \mu \mathrm{M}$. The antibacterial activity could be presented as follows: $\mathbf{5}>\mathbf{3}>\mathbf{7}>\mathbf{6}>\mathbf{9}>\mathbf{1}>\mathbf{1 0}>\mathbf{4}>\mathbf{2}>\mathbf{8}$, but higher than the tested commercial drugs streptomycin and ampicillin. The most resistant bacteria to these compounds were Listeria monocytogenes, while the most susceptible bacteria were Bacillus cereus and Staphylococcus aureus. The obtained results are in agreement with Isloor et al., ${ }^{26}$ who have synthesized 1,2,4-triazole-3(4H)-thione derivatives and reported higher antibacterial activity for the compounds with p-substitutions.

\subsection{Antifungal activity}

All the triazole-sucrose derivatives showed antifungal activity (also evaluated by a microdilution method) with MICs from 0.6 to $26 \mu \mathrm{M}$ and minimal fungicidal concentrations (MFCs) ranging between 2.2 and $39 \mu \mathrm{M}$ (Table 2 ). The antifungal activity could be presented as follows: $\mathbf{3}>\mathbf{1}>\mathbf{5}>\mathbf{7}>\mathbf{9}>\mathbf{4}>\mathbf{2}>\mathbf{6}>\mathbf{8}>\mathbf{1 0}$ and once more, higher than the tested standards, bifonazole and ketoconazole. The highest activity was verified for Trichoderma viride, while Aspergillus fumigatus was the most resistant fungi.

Fungi were in general more sensitive than bacterial species. The antifungal activity exhibited by many potent antifungal agents has

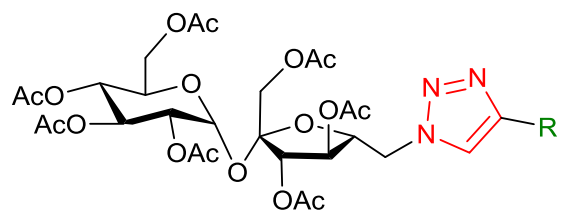

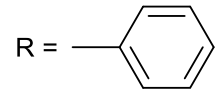

1

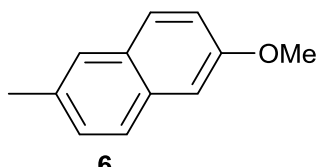

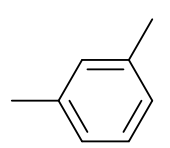

2

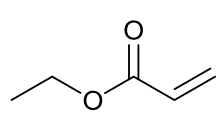

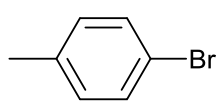

3<smiles>C=C(C)C(=O)OCC</smiles>

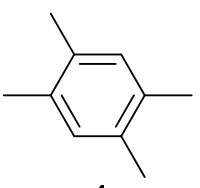

4

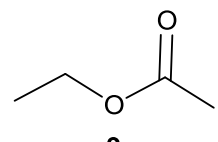

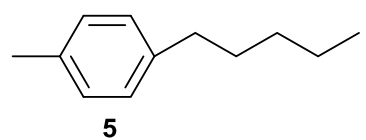

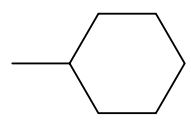

10

Fig. 1. General structure and library of the synthesized $1-\left(1^{\prime}, 2,3,3^{\prime}, 4,4^{\prime}, 6\right.$-hepta-O-acetyl-6'-deoxy-sucros-6 $6^{\prime}$-yl $)-1,2,3-$ triazoles. ${ }^{39}$ 
Table 1

Antibacterial activity of the synthesized 1,2,3-triazole-sucrose derivatives

\begin{tabular}{|c|c|c|c|c|c|c|c|c|c|}
\hline Compound & $\mu \mathrm{M}$ & $\begin{array}{l}\text { Staphylococcus } \\
\text { aureus }\end{array}$ & $\begin{array}{l}\text { Bacillus } \\
\text { cereus }\end{array}$ & $\begin{array}{l}\text { Micrococcus } \\
\text { flavus }\end{array}$ & $\begin{array}{l}\text { Listeria } \\
\text { monocytogenes }\end{array}$ & $\begin{array}{l}\text { Pseudomonas } \\
\text { aeruginosa }\end{array}$ & $\begin{array}{l}\text { Salmonella } \\
\text { typhimurium }\end{array}$ & $\begin{array}{l}\text { Escherichia } \\
\text { coli }\end{array}$ & $\begin{array}{l}\text { Enterobacter } \\
\text { cloacae }\end{array}$ \\
\hline \multirow[t]{2}{*}{1} & MIC & $9.80 \pm 0.02^{\mathrm{a}}$ & $2.60 \pm 0.01^{c}$ & $9.80 \pm 0.02^{\mathrm{b}, \mathrm{c}}$ & $9.80 \pm 0.02^{c}$ & $9.80 \pm 0.01^{c}$ & $5.20 \pm 0.02^{\mathrm{b}, \mathrm{c}}$ & $9.80 \pm 0.07^{b}$ & $13.1 \pm 0.2^{\mathrm{a}}$ \\
\hline & MBC & $19.6 \pm 0.1^{\mathrm{b}}$ & $19.6 \pm 0.1^{\mathrm{a}}$ & $19.6 \pm 0.1^{\mathrm{b}}$ & $19.6 \pm 0.1^{\mathrm{b}}$ & $19.6 \pm 0.1^{\mathrm{b}}$ & $19.6 \pm 0.1^{\mathrm{a}}$ & $19.6 \pm 0.1^{\mathrm{a}}$ & $19.6 \pm 0.1^{b}$ \\
\hline \multirow[t]{2}{*}{2} & MIC & $9.70 \pm 0.01^{a}$ & $5.2 \pm 0.02^{b}$ & $12.9 \pm 0.2^{b}$ & $9.70 \pm 0.01^{c}$ & $12.9 \pm 0.2^{b}$ & $12.9 \pm 0.1^{\mathrm{a}}$ & $12.9 \pm 0.1^{\mathrm{a}}$ & $9.70 \pm 0.02^{b}$ \\
\hline & MBC & $19.4 \pm 0.1^{\mathrm{b}}$ & $19.4 \pm 0.2^{\mathrm{a}}$ & $19.4 \pm 0.2^{\mathrm{b}}$ & $19.4 \pm 0.1^{\mathrm{b}}$ & $19.4 \pm 0.1^{\mathrm{b}}$ & $19.4 \pm 0.1^{\mathrm{a}}$ & $19.4 \pm 0.1^{\mathrm{a}}$ & $19.4 \pm 0.1^{b}$ \\
\hline \multirow[t]{2}{*}{3} & MIC & $8.90 \pm 0.02^{\mathrm{a}}$ & $4.80 \pm 0.03^{b}$ & $8.90 \pm 0.01^{b, c}$ & $4.80 \pm 0.02^{\mathrm{d}}$ & $4.80 \pm 0.02^{\mathrm{d}}$ & $2.40 \pm 0.01^{\mathrm{c}}$ & $4.80 \pm 0.03^{c}$ & $1.20 \pm 0.07^{\mathrm{d}}$ \\
\hline & MBC & $17.8 \pm 0.2^{\mathrm{b}}$ & $17.8 \pm 0.1^{\mathrm{a}}$ & $17.8 \pm 0.1^{c}$ & $17.8 \pm 0.2^{c}$ & $17.8 \pm 0.1^{\mathrm{b}}$ & $4.80 \pm 0.02^{\mathrm{d}}$ & $8.90 \pm 0.02^{b}$ & $8.90 \pm 0.03^{c}$ \\
\hline \multirow[t]{2}{*}{4} & MIC & $4.90 \pm 0.02^{\mathrm{b}}$ & $12.40 \pm 0.07^{\mathrm{a}}$ & $12.4 \pm 0.1^{\mathrm{b}}$ & $12.4 \pm 0.1^{\mathrm{b}}$ & $12.4 \pm 0.1^{\mathrm{b}}$ & $9.30 \pm 0.02^{\mathrm{a}, \mathrm{b}}$ & $12.4 \pm 0.1^{\mathrm{a}}$ & $9.30 \pm 0.02^{\mathrm{b}}$ \\
\hline & MBC & $18.6 \pm 0.1^{b}$ & $18.60 \pm 0.03^{\mathrm{a}}$ & $18.60 \pm 0.01^{b}$ & $18.60 \pm 0.07^{\mathrm{b}, \mathrm{c}}$ & $18.6 \pm 0.2^{b}$ & $18.6 \pm 0.2^{\mathrm{a}}$ & $18.60 \pm 0.07^{a}$ & $18.6 \pm 0.1^{b}$ \\
\hline \multirow[t]{2}{*}{5} & MIC & $1.10 \pm 0.01^{\mathrm{c}}$ & $1.10 \pm 0.01^{d}$ & $4.40 \pm 0.02^{\mathrm{d}}$ & $4.40 \pm 0.01^{\mathrm{d}}$ & $1.10 \pm 0.01^{\mathrm{e}}$ & $1.10 \pm 0.01^{\mathrm{d}}$ & $4.40 \pm 0.03^{c}$ & $1.10 \pm 0.01^{\mathrm{d}}$ \\
\hline & MBC & $2.20 \pm 0.07^{d}$ & $4.40 \pm 0.02^{c}$ & $8.40 \pm 0.02^{\mathrm{d}}$ & $33.6 \pm 0.1^{\mathrm{a}}$ & $8.4 \pm 0.1^{c, d}$ & $4.40 \pm 0.01^{\mathrm{d}}$ & $8.40 \pm 0.07^{b}$ & $1.70 \pm 0.01^{\mathrm{d}}$ \\
\hline \multirow[t]{2}{*}{6} & MIC & $8.90 \pm 0.02^{\mathrm{a}}$ & $4.80 \pm 0.02^{\mathrm{b}}$ & $11.90 \pm 0.01^{b, c}$ & $11.9 \pm 0.2^{\mathrm{b}}$ & $11.90 \pm 0.03^{b}$ & $8.90 \pm 0.01^{\mathrm{a}, \mathrm{b}}$ & $11.90 \pm 0.01^{\mathrm{a}}$ & $4.80 \pm 0.04^{c}$ \\
\hline & MBC & $17.8 \pm 0.2^{\mathrm{b}}$ & $17.8 \pm 0.1^{\mathrm{a}}$ & $17.8 \pm 0.2^{c}$ & $17.8 \pm 0.1^{\mathrm{c}}$ & $17.80 \pm 0.03^{b}$ & $17.8 \pm 0.2^{\mathrm{b}}$ & $17.80 \pm 0.07^{a}$ & $35.60 \pm 0.02^{a}$ \\
\hline \multirow[t]{2}{*}{7} & MIC & $5.20 \pm 0.07^{b}$ & $5.20 \pm 0.03^{b}$ & $7.80 \pm 0.02^{c}$ & $9.70 \pm 0.02^{c}$ & $9.7 \pm 0.02^{c}$ & $2.60 \pm 0.02^{c}$ & $9.70 \pm 0.02^{\mathrm{b}}$ & $5.20 \pm 0.01^{c}$ \\
\hline & MBC & $9.70 \pm 0.02^{c}$ & $9.70 \pm 0.02^{\mathrm{b}}$ & $9.70 \pm 0.01^{\mathrm{d}}$ & $19.4 \pm 0.1^{\mathrm{b}}$ & $19.40 \pm 0.01^{b}$ & $9.70 \pm 0.01^{c}$ & $19.40 \pm 0.01^{\mathrm{a}}$ & $19.40 \pm 0.03^{b}$ \\
\hline \multirow[t]{2}{*}{8} & MIC & $9.50 \pm 0.01^{\mathrm{a}}$ & $5.10 \pm 0.03^{b}$ & $19.0 \pm 0.1^{\mathrm{a}}$ & $25.4 \pm 0.1^{\mathrm{a}}$ & $38.0 \pm 0.1^{\mathrm{a}}$ & $9.50 \pm 0.07^{\mathrm{a}, \mathrm{b}}$ & $12.7 \pm 0.1^{\mathrm{a}}$ & $5.10 \pm 0.01^{c}$ \\
\hline & MBC & $38.0 \pm 0.1^{\mathrm{a}}$ & $19.0 \pm 0.1^{\mathrm{a}}$ & $38.0 \pm 0.2^{\mathrm{a}}$ & $38.0 \pm 0.2^{\mathrm{a}}$ & $57.2 \pm 0.1^{\mathrm{a}}$ & $19.0 \pm 0.1^{\mathrm{a}}$ & $19.0 \pm 0.1^{\mathrm{a}}$ & $19.0 \pm 0.1^{b}$ \\
\hline \multirow[t]{2}{*}{9} & MIC & $9.90 \pm 0.02^{\mathrm{a}}$ & $1.30 \pm 0.07^{d}$ & $9.90 \pm 0.02^{\mathrm{b}, \mathrm{c}}$ & $9.90 \pm 0.01^{c}$ & $9.90 \pm 0.01^{c}$ & $1.30 \pm 0.05^{c}$ & $9.90 \pm 0.02^{b}$ & $5.20 \pm 0.03^{c}$ \\
\hline & MBC & $19.8 \pm 0.1^{b}$ & $19.8 \pm 0.2^{\mathrm{a}}$ & $19.8 \pm 0.2^{\mathrm{b}}$ & $19.8 \pm 0.1^{b}$ & $19.8 \pm 0.1^{b}$ & $19.8 \pm 0.1^{\mathrm{a}}$ & $19.8 \pm 0.1^{\mathrm{a}}$ & $19.8 \pm 0.1^{b}$ \\
\hline \multirow[t]{2}{*}{10} & MIC & $9.80 \pm 0.01^{\mathrm{a}}$ & $5.20 \pm 0.01^{\mathrm{b}}$ & $13.0 \pm 0.07^{b}$ & $9.80 \pm 0.01^{\mathrm{c}}$ & $9.80 \pm 0.01^{c}$ & $5.20 \pm 0.07^{b, c}$ & $13.0 \pm 0.2^{\mathrm{a}}$ & $9.80 \pm 0.07^{b}$ \\
\hline & MBC & $19.6 \pm 0.1^{\mathrm{b}}$ & $19.6 \pm 0.1^{\mathrm{a}}$ & $19.6 \pm 0.1^{b}$ & $19.6 \pm 0.2^{\mathrm{b}}$ & $19.6 \pm 0.1^{\mathrm{b}}$ & $19.6 \pm 0.1^{\mathrm{a}}$ & $19.6 \pm 0.1^{\mathrm{a}}$ & $19.6 \pm 0.1^{b}$ \\
\hline \multirow[t]{2}{*}{ Streptomy-cin } & MIC & $20 \pm 1$ & $45.6 \pm 1$ & $86 \pm 1$ & $86 \pm 1$ & $86 \pm 1$ & $86 \pm 1$ & $86 \pm 1$ & $132 \pm 1$ \\
\hline & MBC & $46 \pm 1$ & $86 \pm 1$ & $172 \pm 1$ & $172 \pm 1$ & $172 \pm 1$ & $172 \pm 1$ & $172 \pm 1$ & $264 \pm 2$ \\
\hline \multirow[t]{2}{*}{ Ampi-cillin } & MIC & $116 \pm 1$ & $116 \pm 1$ & $116 \pm 1$ & $172 \pm 1$ & $344 \pm 2$ & $172 \pm 1$ & $116 \pm 1$ & $172 \pm 1$ \\
\hline & MBC & $172 \pm 1$ & $172 \pm 1$ & $172 \pm 1$ & $228 \pm 2$ & $576 \pm 2$ & $228 \pm 2$ & $228 \pm 2$ & $344 \pm 2$ \\
\hline
\end{tabular}

MIC, minimum inhibitory concentration; MBC, minimum bactericidal concentration.

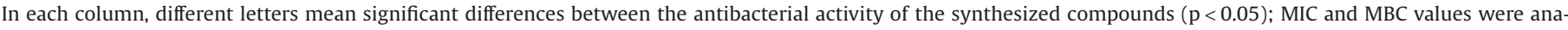
lyzed separately.

been attributed to the presence of triazole ring system; one of the major examples containing triazole ring is fluconazole. ${ }^{8}$

According to the obtained results, it is obvious that the derivatives 5 (with 4-pentylphenyl substituent) showed the best antibacterial activity, while $\mathbf{3}$ (with 4-bromophenyl substituent) possessed the best antifungal activity.

The results showed that the growth of the various types of bacteria tested responded differently to the synthetic compounds. This indicates that it may have different modes of action on the different species or that the metabolism of some bacteria is able to overcome the effect of the compound or adapt to it. It is known that Gram positive bacterial species are more susceptible to antimicrobial agents than Gram negative bacteria, and that fungi are more susceptible than bacteria in general. ${ }^{42,43}$ Thus, the susceptibility of microorganisms to external agents depends not only on their properties, but also on the microorganism itself.

Table 2

Antifungal activity of the synthesized 1,2,3-triazole-sucrose derivatives

\begin{tabular}{|c|c|c|c|c|c|c|c|c|c|}
\hline Compound & $\mu \mathrm{M}$ & $\begin{array}{l}\text { Aspergillus } \\
\text { fumigatus }\end{array}$ & $\begin{array}{l}\text { Aspergillus } \\
\text { versicolor }\end{array}$ & $\begin{array}{l}\text { Aspergillus } \\
\text { ochraceus }\end{array}$ & $\begin{array}{l}\text { Aspergillus } \\
\text { niger }\end{array}$ & $\begin{array}{l}\text { Trichoderma } \\
\text { viride }\end{array}$ & $\begin{array}{l}\text { Penicillium } \\
\text { funiculosum }\end{array}$ & $\begin{array}{l}\text { Penicillium } \\
\text { ochrochloron }\end{array}$ & $\begin{array}{l}\text { Penicillium } \\
\text { verrucosum }\end{array}$ \\
\hline \multirow[t]{2}{*}{1} & MIC & $9.80 \pm 0.01^{c}$ & $1.30 \pm 0.01^{\mathrm{c}}$ & $0.65 \pm 0.02^{\mathrm{d}}$ & $2.60 \pm 0.01^{\mathrm{c}}$ & $1.30 \pm 0.01^{c}$ & $5.20 \pm 0.02^{\mathrm{c}, \mathrm{d}}$ & $5.20 \pm 0.02^{c}$ & $5.20 \pm 0.01^{d}$ \\
\hline & MFC & $19.6 \pm 0.1^{\mathrm{b}}$ & $2.60 \pm 0.02^{\mathrm{d}}$ & $2.60 \pm 0.01^{\mathrm{c}}$ & $5.20 \pm 0.02^{c, d}$ & $2.60 \pm 0.02^{\mathrm{c}, \mathrm{d}}$ & $9.80 \pm 0.01^{\mathrm{c}}$ & $9.80 \pm 0.01^{c}$ & $9.80 \pm 0.02^{\mathrm{b}}$ \\
\hline \multirow[t]{2}{*}{2} & MIC & $9.70 \pm 0.01^{c}$ & $9.70 \pm 0.03^{a}$ & $5.20 \pm 0.01^{\mathrm{a}}$ & $5.20 \pm 0.02^{\mathrm{b}}$ & $2.60 \pm 0.01^{\mathrm{b}}$ & $9.70 \pm 0.01^{\mathrm{c}}$ & $9.70 \pm 0.01^{b}$ & $25.8 \pm 0.1^{\mathrm{a}}$ \\
\hline & MFC & $38.8 \pm 0.1^{\mathrm{a}}$ & $19.4 \pm 0.1^{b}$ & $9.70 \pm 0.02^{\mathrm{a}}$ & $9.70 \pm 0.02^{c}$ & $5.20 \pm 0.02^{\mathrm{a}, \mathrm{b}}$ & $19.4 \pm 0.1^{\mathrm{b}}$ & $19.4 \pm 0.1^{\mathrm{b}}$ & $38.8 \pm 0.1^{\mathrm{a}}$ \\
\hline \multirow[t]{2}{*}{3} & MIC & $4.80 \pm 0.10^{d}$ & $1.20 \pm 0.01^{\mathrm{c}}$ & $1.20 \pm 0.01^{\mathrm{c}}$ & $2.40 \pm 0.02^{c}$ & $0.60 \pm 0.01^{\mathrm{d}}$ & $1.20 \pm 0.01^{\mathrm{e}}$ & $2.40 \pm 0.01^{\mathrm{d}}$ & $2.40 \pm 0.01^{\mathrm{d}}$ \\
\hline & MFC & $8.90 \pm 0.01^{c}$ & $4.80 \pm 0.02^{c}$ & $2.40 \pm 0.01^{\mathrm{c}}$ & $4.80 \pm 0.01^{\mathrm{d}}$ & $1.20 \pm 0.01^{\mathrm{d}}$ & $4.80 \pm 0.02^{d}$ & $4.80 \pm 0.03^{d}$ & $4.80 \pm 0.01^{c}$ \\
\hline \multirow[t]{2}{*}{4} & MIC & $24.8 \pm 0.1^{\mathrm{b}}$ & $9.30 \pm 0.01^{a}$ & $4.90 \pm 0.01^{\mathrm{a}}$ & $4.90 \pm 0.01^{b}$ & $3.70 \pm 0.01^{\mathrm{a}, \mathrm{b}}$ & $9.30 \pm 0.02^{c}$ & $9.30 \pm 0.01^{b}$ & $9.30 \pm 0.07^{b}$ \\
\hline & MFC & $37.2 \pm 0.1^{\mathrm{a}}$ & $18.6 \pm 0.1^{\mathrm{b}}$ & $9.30 \pm 0.01^{\mathrm{a}}$ & $18.6 \pm 0.1^{b}$ & $4.90 \pm 0.01^{\mathrm{b}}$ & $18.6 \pm 0.1^{b}$ & $37.2 \pm 0.1^{\mathrm{a}}$ & $37.2 \pm 0.1^{\mathrm{a}}$ \\
\hline \multirow[t]{2}{*}{5} & MIC & $8.40 \pm 0.01^{\mathrm{b}, \mathrm{c}}$ & $3.30 \pm 0.03^{b}$ & $2.20 \pm 0.01^{b}$ & $4.40 \pm 0.01^{b}$ & $1.10 \pm 0.01^{\mathrm{c}, \mathrm{d}}$ & $4.40 \pm 0.01^{\mathrm{d}}$ & $8.40 \pm 0.01^{b}$ & $6.60 \pm 0.01^{c}$ \\
\hline & MFC & $16.8 \pm 0.1^{b}$ & $4.40 \pm 0.01^{c}$ & $4.40 \pm 0.02^{b}$ & $8.40 \pm 0.01^{c}$ & $2.20 \pm 0.02^{\mathrm{b}, \mathrm{c}}$ & $8.40 \pm 0.02^{c}$ & $16.8 \pm 0.1^{\mathrm{b}}$ & $8.40 \pm 0.01^{b}$ \\
\hline \multirow[t]{2}{*}{6} & MIC & $23.8 \pm 0.1^{\mathrm{b}}$ & $8.90 \pm 0.01^{a}$ & $4.80 \pm 0.02^{\mathrm{a}}$ & $8.90 \pm 0.02^{\mathrm{a}}$ & $4.80 \pm 0.01^{\mathrm{a}}$ & $23.8 \pm 0.1^{\mathrm{a}}$ & $23.8 \pm 0.1^{\mathrm{a}}$ & $23.8 \pm 0.1^{\mathrm{a}}$ \\
\hline & MFC & $35.7 \pm 0.1^{\mathrm{a}}$ & $17.8 \pm 0.1^{\mathrm{b}}$ & $8.90 \pm 0.01^{\mathrm{a}}$ & $17.8 \pm 0.1^{b}$ & $8.90 \pm 0.01^{\mathrm{a}}$ & $35.7 \pm 0.2^{\mathrm{a}}$ & $35.7 \pm 0.2^{\mathrm{a}}$ & $35.7 \pm 0.1^{\mathrm{a}}$ \\
\hline \multirow[t]{2}{*}{7} & MIC & $5.20 \pm 0.07^{d}$ & $2.60 \pm 0.02^{b, c}$ & $2.60 \pm 0.01^{b}$ & $2.60 \pm 0.01^{c}$ & $1.30 \pm 0.01^{c, d}$ & $2.60 \pm 0.02^{\mathrm{d}, \mathrm{e}}$ & $5.20 \pm 0.02^{\mathrm{b}, \mathrm{c}}$ & $5.20 \pm 0.02^{c}$ \\
\hline & MFC & $19.4 \pm 0.1^{b}$ & $5.20 \pm 0.07^{c}$ & $5.20 \pm 0.02^{b}$ & $9.70 \pm 0.01^{c}$ & $2.60 \pm 0.01^{\mathrm{b}, \mathrm{c}}$ & $9.70 \pm 0.01^{\mathrm{c}}$ & $9.70 \pm 0.01^{c}$ & $9.70 \pm 0.01^{b}$ \\
\hline \multirow[t]{2}{*}{8} & MIC & $25.4 \pm 0.1^{\mathrm{b}}$ & $9.50 \pm 0.02^{\mathrm{a}}$ & $5.10 \pm 0.01^{\mathrm{a}}$ & $5.10 \pm 0.02^{b}$ & $5.10 \pm 0.02^{\mathrm{a}}$ & $9.50 \pm 0.01^{\mathrm{c}}$ & $9.50 \pm 0.01^{b}$ & $19.0 \pm 0.1^{b}$ \\
\hline & MFC & $38.0 \pm 0.2^{\mathrm{a}}$ & $38.0 \pm 0.1^{\mathrm{a}}$ & $9.50 \pm 0.02^{\mathrm{a}}$ & $19.0 \pm 0.1^{b}$ & $9.50 \pm 0.01^{\mathrm{a}}$ & $19.0 \pm 0.1^{b}$ & $38.0 \pm 0.1^{\mathrm{a}}$ & $38.0 \pm 0.1^{\mathrm{a}}$ \\
\hline \multirow[t]{2}{*}{9} & MIC & $9.90 \pm 0.03^{c}$ & $1.30 \pm 0.01^{\mathrm{c}}$ & $2.60 \pm 0.02^{b}$ & $2.60 \pm 0.01^{\mathrm{c}}$ & $2.00 \pm 0.02^{\mathrm{b}}$ & $2.60 \pm 0.01^{\mathrm{d}, \mathrm{e}}$ & $2.60 \pm 0.01^{\mathrm{d}}$ & $5.20 \pm 0.01^{c}$ \\
\hline & MFC & $19.8 \pm 0.1^{b}$ & $2.60 \pm 0.07^{c, d}$ & $5.20 \pm 0.01^{b}$ & $9.90 \pm 0.01^{\mathrm{c}}$ & $2.60 \pm 0.01^{b, c}$ & $5.20 \pm 0.02^{c, d}$ & $9.90 \pm 0.02^{c}$ & $9.90 \pm 0.01^{b}$ \\
\hline \multirow[t]{2}{*}{10} & MIC & $19.6 \pm 0.1^{\mathrm{b}, \mathrm{c}}$ & $9.80 \pm 0.01^{\mathrm{a}}$ & $5.20 \pm 0.01^{\mathrm{a}}$ & $9.80 \pm 0.02^{\mathrm{a}}$ & $3.90 \pm 0.02^{\mathrm{b}}$ & $9.80 \pm 0.01^{c}$ & $19.6 \pm 0.1^{\mathrm{a}}$ & $26.0 \pm 0.1^{\mathrm{a}}$ \\
\hline & MFC & $39.2 \pm 0.1^{\mathrm{a}}$ & $39.2 \pm 0.1^{\mathrm{a}}$ & $9.80 \pm 0.03^{a}$ & $39.2 \pm 0.1^{\mathrm{a}}$ & $5.20 \pm 0.01^{\mathrm{ab}}$ & $19.6 \pm 0.1^{\mathrm{b}}$ & $39.2 \pm 0.1^{\mathrm{a}}$ & $39.2 \pm 0.1^{a}$ \\
\hline \multirow[t]{2}{*}{ Ketoco-nazole } & MIC & $380 \pm 1$ & $380 \pm 1$ & $285 \pm 1$ & $380 \pm 1$ & $1900 \pm 4$ & $380 \pm 1$ & $1900 \pm 4$ & $2850 \pm 4$ \\
\hline & MFC & $950 \pm 3$ & $950 \pm 3$ & $380 \pm 1$ & $950 \pm 3$ & $2850 \pm 4$ & $950 \pm 3$ & $2850 \pm 4$ & $3800 \pm 4$ \\
\hline \multirow[t]{2}{*}{ Bifo-nazole } & MIC & $480 \pm 1$ & $320 \pm 1$ & $480 \pm 1$ & $480 \pm 1$ & $480 \pm 1$ & $640 \pm 2$ & $640 \pm 1$ & $640 \pm 1$ \\
\hline & MFC & $640 \pm 2$ & $640 \pm 2$ & $640 \pm 2$ & $640 \pm 2$ & $640 \pm 2$ & $800 \pm 2$ & $800 \pm 2$ & $960 \pm 3$ \\
\hline
\end{tabular}

MIC, minimum inhibitory concentration; MFC, minimum fungicidal concentration.

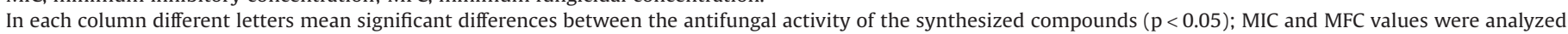
separately. 
Table 3

Cytotoxicity of the synthesized 1,2,3-triazole-sucrose derivatives ( $\mathrm{GI}_{50}$ values in $\mu \mathrm{M}$ )

\begin{tabular}{|c|c|c|c|c|c|}
\hline & $\begin{array}{l}\text { MCF7 } \\
\text { (breast carcinoma) }\end{array}$ & $\begin{array}{l}\mathrm{NCI}-\mathrm{H} 460 \\
\text { (non-small lung carcinoma) }\end{array}$ & $\begin{array}{l}\text { HeLa } \\
\text { (cervical carcinoma) }\end{array}$ & $\begin{array}{l}\text { HepG2 } \\
\text { (hepatocellular carcinoma) }\end{array}$ & $\begin{array}{l}\text { PLP2 } \\
\text { (liver primary culture) }\end{array}$ \\
\hline 1 & $228 \pm 12^{b}$ & $210 \pm 7^{b}$ & $269 \pm 18^{a}$ & $280 \pm 9^{a}$ & $>500$ \\
\hline 3 & $>500$ & $>500$ & $>500$ & $195 \pm 8$ & $>500$ \\
\hline 7 & $318 \pm 4$ & $>500$ & $>500$ & $>500$ & $>500$ \\
\hline 10 & $>500$ & $>500$ & $>500$ & $375 \pm 15$ & $>500$ \\
\hline Ellipticine & $3.7 \pm 0.2$ & $8.0 \pm 0.2$ & $4.75 \pm 0.05$ & $13 \pm 1$ & $8.6 \pm 0.1$ \\
\hline
\end{tabular}

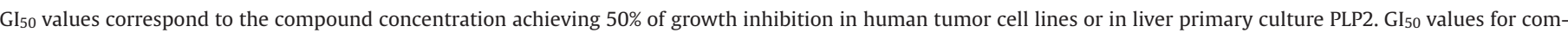
pounds $2,4-6,8$ and 9 were $>500 \mu \mathrm{M}$.

For compound 1, different letters mean significant differences for different cell lines $(\mathrm{p}<0.05)$.

\subsection{Cytotoxicity}

The cytotoxicity of the compounds was evaluated, through the Sulforhodamine B assay, against four human tumor cell lines (breast - MCF7, non-small cell lung - NCI-H460, cervical - HeLa and hepatocellular - HepG2, carcinomas), and in a porcine liver primary cell culture (PLP2), established by us. Compound 1 inhibited the growth of all the tested tumor cell lines (Table 3 ). The cytotoxicity showed by this compound may be due to the presence of 4-phenyl1,2,3-triazole in the end group. This group was also reported to be effective in suppressing pancreatic cancer cells in vitro growth. ${ }^{2}$

Compounds $\mathbf{3}$ and $\mathbf{1 0}$ (similar structure to compound 1, with a 4-bromophenyl and cyclohexyl substituents, respectively) only inhibited the HepG2 cell line, while compound 7 (with an acryloxymethyl substituent) showed moderate activity against the MCF7 cell line. Compounds 2, 4-6, 8 and 9 were not active against any of the tested cell lines, at the maximum concentration tested $(500 \mu \mathrm{M})$.

Up to the same concentration, none of the tested compounds showed toxicity toward the non-tumor liver primary culture (PLP2).

\section{Conclusions}

In summary, a small library of sucrose 1,2,3-triazoles has been created and screened for antibacterial, antifungal and cytotoxic activities in order to identify lead compounds for the pharmacology. The experimental results showed that compound $\mathbf{5}$ (with 4-pentylphenyl substituent) had the highest antibacterial activity, while compound $\mathbf{3}$ (with 4-bromophenyl substituent) was the strongest antifungal agent. Also, the compounds showed a moderate cytotoxicity against some of the tested tumor cell lines, but without toxicity for non-tumor liver cells.

\section{Materials and methods}

\subsection{Standards and reagents}

Ampicillin, bifonazole and ketoconazole were purchased by Panfarma (Belgrade, Serbia), Srbolek (Belgrade, Serbia) and Zorkapharma (Šabac, Serbia), respectively. Fetal bovine serum (FBS), L-glutamine, Hank's balanced salt solution (HBSS), trypsin-EDTA (ethylenediaminetetraacetic acid), penicillin/streptomycin solution (100 U/mL and $100 \mathrm{mg} / \mathrm{mL}$, respectively), and RPMI-1640 were from Hyclone (Logan, USA). Streptomycin, acetic acid, ellipticine, sulforhodamine B (SRB), trypan blue, trichloroacetic acid (TCA) and Tris were purchased from Sigma Chemical Co. (Saint Louis, USA). Water was treated in a Milli-Q water purification system (TGI Pure Water Systems, USA).

\subsection{Chemistry}

Compounds 1-10 were synthesized as previously described ${ }^{39}$ using microwave irradiation and isolated by flash column chromatography in pure form. Their structures and purity were confirmed by common spectroscopic techniques (IR, NMR), MALDI-TOF, m.p. and $[\alpha]_{D}$.

Stock solutions of the compounds were prepared in 5\% DMSO and kept at $-20^{\circ} \mathrm{C}$. Prior to the assays, appropriate dilutions were prepared.

\subsection{Antimicrobial activity}

\subsubsection{Antibacterial activity}

The Gram-positive bacteria S. aureus (ATCC 6538), B. cereus (clinical isolate), Micrococcus flavus (ATCC 10240) and Listeria monocytogenes (NCTC 7973), and the Gram-negative bacteria Pseudomonas aeruginosa (ATCC 27853), Salmonella typhimurium (ATCC 13311), Escherichia coli (ATCC 35210), and Enterobacter cloacae (human isolate) were used. The antibacterial assay was carried out by a microdilution method. ${ }^{44,45}$ The bacterial suspensions were adjusted with sterile saline to a concentration of $1.0 \times 10^{5} \mathrm{CFU} / \mathrm{mL}$. Compound solutions were added to the Tryptic Soy broth (TSB) medium $(100 \mu \mathrm{L})$ with bacterial inoculum $\left(1.0 \times 10^{4} \mathrm{CFU}\right.$ per well). The lowest concentrations without visible growth (at the binocular microscope) were defined as concentrations that completely inhibited bacterial growth (MICs). The MICs obtained from the susceptibility testing of various bacteria to tested extracts were determined also by a colorimetric microbial viability assay based on reduction of an INT ((p-iodonitrotetrazolium violet) [2-(4iodophenyl)-3-(4-nitrphenyl)-5-phenyltetrazolium chloride; Sigma]) color and compared with positive control for each bacterial strains. The MBCs were determined by serial sub-cultivation of $2 \mu \mathrm{L}$ into microtiter plates containing $100 \mu \mathrm{L}$ of broth per well and further incubation for $24 \mathrm{~h}$. The lowest concentration with no visible growth was defined as the MBC, indicating 99.5\% killing of the original inoculum. The optical density of each well was measured at a wavelength of $655 \mathrm{~nm}$ by Microplate manager 4.0 (Bio-Rad Laboratories) and compared with a blank (broth medium plus diluted extracts) and the positive control. Streptomycin and ampicillin were used as positive controls. Five percent DMSO was used as a negative control.

\subsubsection{Antifungal activity}

Aspergillus fumigatus (human isolate), A. versicolor (ATCC 11730), A. ochraceus (ATCC 12066), A. niger (ATCC 6275), Trichoderma viride (IAM 5061), Penicillium funiculosum (ATCC 36839), P. ochrochloron (ATCC 9112) and P. verrucosum var. cyclopium (food isolate) were used. In order to investigate the antifungal activity of the compounds, a modified microdilution technique was used. ${ }^{46}$ The fungal 
spores were washed from the surface of agar plates with sterile $0.85 \%$ saline containing $0.1 \%$ Tween $80(\mathrm{v} / \mathrm{v})$ and spore suspension was adjusted with sterile saline to a concentration of $1.0 \times 10^{5}$. Compound solutions were added to the broth Malt medium with inoculum. The lowest concentrations without visible growth (at the binocular microscope) were defined as MICs. The fungicidal concentrations (MFCs) were determined by serial subcultivation of a $2 \mu \mathrm{L}$ of tested compounds dissolved in medium and incubated for $72 \mathrm{~h}$ at $28^{\circ} \mathrm{C}$. The lowest concentration with no visible growth was defined as MFC indicating 99.5\% killing of the original inoculum. DMSO was used as a negative control, and commercial fungicides, bifonazole and ketoconazole were used as positive controls. Five percent DMSO was used as a negative control.

\subsection{Cytotoxic activity}

\subsubsection{Cytotoxicity in human tumor cell lines}

Four human tumor cell lines were used: MCF-7 (breast adenocarcinoma), $\mathrm{NCI}-\mathrm{H} 460$ (non-small cell lung carcinoma), HeLa (cervical carcinoma) and HepG2 (hepatocellular carcinoma) from DSMZ (Leibniz-Institut DSMZ-Deutsche Sammlung von Mikroorganismen und Zellkulturen $\mathrm{GmbH}$ ). Cells were routinely maintained as adherent cell cultures in RPMI-1640 medium containing 10\% heatinactivated FBS and $2 \mathrm{mM}$ glutamine at $37{ }^{\circ} \mathrm{C}$, in a humidified air incubator containing $5 \% \mathrm{CO}_{2}$. Each cell line was plated at an appropriate density $\left(7.5 \times 10^{3}\right.$ cells/well for MCF-7 and NCI-H460 or $1.0 \times 10^{4}$ cells/well for HeLa and HepG2) in 96-well plates and allowed to attach for $24 \mathrm{~h}$. Cells were then treated for $48 \mathrm{~h}$ with various concentrations of the compounds. Following this incubation period, the adherent cells were fixed by adding cold $10 \%$ trichloroacetic acid (TCA, $100 \mu \mathrm{L}$ ) and incubated for $60 \mathrm{~min}$ at $4{ }^{\circ} \mathrm{C}$. Plates were then washed with deionized water and dried; sulforhodamine B (SRB) solution $(0.1 \%$ in $1 \%$ acetic acid, $100 \mu \mathrm{L})$ was then added to each plate well and incubated for $30 \mathrm{~min}$ at room temperature. Unbound SRB was removed by washing with $1 \%$ acetic acid. Plates were air dried, the bound SRB was solubilized with $10 \mathrm{mM}$ Tris $(200 \mu \mathrm{L})$ and the absorbance was measured at $540 \mathrm{~nm}$ in ELX800 Microplate Reader (Bio-Tek Instruments, Inc; Winooski, USA). ${ }^{47}$ The results were expressed in $\mathrm{GI}_{50}$ values (compound concentration that inhibited 50\% of the net cell growth). Ellipticine was used as positive control. The absence of DMSO toxicity was confirmed by treating cells with the maximum concentration of DMSO used in the assays ( $0.25 \%)$.

\subsubsection{Cytotoxicity in a porcine liver primary cell culture}

A cell culture was prepared from a freshly harvested porcine liver obtained from a local slaughter house, and it was designed as PLP2. Briefly, the liver tissues were rinsed in Hank's balanced salt solution containing $100 \mathrm{U} / \mathrm{mL}$ penicillin, $100 \mu \mathrm{g} / \mathrm{mL}$ streptomycin and divided into $1 \times 1 \mathrm{~mm}^{3}$ explants. Some of these explants were placed in $25 \mathrm{~cm}^{2}$ tissue flasks in DMEM medium supplemented with $10 \%$ fetal bovine serum, $2 \mathrm{mM}$ nonessential amino acids and $100 \mathrm{U} / \mathrm{mL}$ penicillin, $100 \mathrm{mg} / \mathrm{mL}$ streptomycin and incubated at $37{ }^{\circ} \mathrm{C}$ with a humidified atmosphere containing $5 \% \mathrm{CO}_{2}$. The medium was changed every 2 days. Cultivation of the cells was continued with direct monitoring every 2-3 days using a phase contrast microscope. Before confluence was reached, cells were subcultured and plated in 96well plates at a density of $1.0 \times 10^{4}$ cells/well, and cultivated in DMEM medium with $10 \%$ FBS, $100 \mathrm{U} / \mathrm{mL}$ penicillin and $100 \mu \mathrm{g} / \mathrm{mL}$ streptomycin. Sulforhodamine B assay was performed according to a procedure previously described. ${ }^{47}$ The results were expressed in $\mathrm{GI}_{50}$ values (compound concentration that inhibited $50 \%$ of the net cell growth). Ellipticine was used as positive control. The absence of DMSO toxicity was confirmed by treating cells with the maximum concentration of DMSO used in the assays (0.25\%).

\subsection{Statistical analysis}

For all the experiments three solutions were prepared from each compound concentration, and all the assays were carried out in triplicate. The results are expressed as mean values and standard deviation (SD). The results were analyzed using one-way analysis of variance (ANOVA) followed by Tukey's HSD Test with $\alpha=0.05$. This analysis was carried out using SPSS v. 22.0 program (IBM Corp., Armonk, NY: USA).

\section{Author contributions}

The manuscript was written through contributions of all authors. All authors have given approval to the final version of the manuscript.

\section{Acknowledgments}

This work has been supported by Fundação para a Ciência e a Tecnologia through grant Nos. PEst-C/EQB/LA0006/2013 and PEstOE/AGR/UI0690/2014. T.M. Potewar and R.C. Calhelha are grateful to Fundação para a Ciência e a Tecnologia for their pos-doctoral Grant Nos. SFRH/ BPD/65173/2009 and SFRH/BPD/68344/2010, respectively. The authors thank to Serbian Ministry of Education, Science and Technological Development for financial support (grant number 173032). The NMR spectrometers are part of The National NMR Facility, supported by Fundação para a Ciência e a Tecnologia (RECI/BBB-BQB/0230/2012).

\section{References}

1. Butler MS, Cooper MA. J Antibiot 2011;64:413-25.

2. He R, Chen YF, Chen YH, Ougolkov AV, Zhang JS, Savoy DN, et al. J Med Chem 2010:53:1347-56.

3. Ziemska J, Rajnisz A, Solecka J. Cent Eur J Biol 2013;8:943-57.

4. Liang L, Astruc D. Coord Chem Rev 2011;255:2933-45.

5. Meldal M, Tornoe CW. Chem Rev 2008;108:2952-3015.

6. Tron GC, Pirali T, Billington RA, Canoniico PL, Sorba G, Genazzani AA. Med Res Rev 2008;28:278-308.

7. Zhou CH, Wang Y. Curr Med Chem 2012;19:239-80.

8. Demirbas N, Ugurluoglu R, Demirbas A. Bioorg Med Chem 2002;10:3717-23.

9. Crucho CIC, Correia-da-Silva P, Petrova KT, Barros MT. Carbohydr Res 2015;402:124-32.

10. Dwek AR. Chem Rev 1996;96:683-720.

11. Aragão-Leoneti V, Campo VL, Gomes AS, Field RA, Carvalho I. Tetrahedron 2010;66:9475-92.

12. Perion R, Ferrieres V, Garcia-Moreno MI, Mellet CO, Duval R, Fernandez JMG, et al. Tetrahedron 2005;61:9118-28.

13. Dedola S, Hughes DL, Nepogodiev SA, Rejzek M, Field RA. Carbohydr Res 2010;345:1123-34

14. Castro S, Cherney EC, Snyder NL, Peczuh MW. Carbohydr Res 2007;342:1366-72.

15. Rjabova J, Rjabovs V, Vargas AJM, Clavijo EM, Turks M. Cent Eur J Chem 2012;10:386-94.

16. Carvalho I, Andrade P, Campo VL, Guedes PMM, Sesti-Costa R, Silva JS, et al. Bioorg Med Chem 2010:18:2412-27.

17. Bokor E, Docsa T, Gergely P, Somsak L. Bioorg Med Chem 2010;18:1171-80.

18. Slamova K, Marhol P, Bezouska K, Lindkvist L, Hansen SG, Kren V, et al. Bioorg Med Chem Lett 2010;20:4263-5.

19. Singh BK, Kumar YA, Kumar B, Gaikwad A, Sinha SK, Chaturvedi V, et al. Carbohydr Res 2008;343:1153-62.

20. Ortega-Munoz M, Perez-Balderas F, Morales-Sanfrutos J, Hernandez-Mateo F, Isac-Garcia J, Santoyo-Gonzalez F. Eur J Org Chem 2009;15:2454-73.

21. Krim J, Taourirte M, Engels JW. Molecules 2012;17:179-90.

22. Reddy LVR, Reddy PV, Mishra NN, Shukla PK, Yadav G, Srivastava R, et al. Carbohydr Res 2010;345:1515-21.

23. Xavier NM, Goulart M, Neves A, Justino J, Chambert S, Rauter AP, et al. Bioorg Med Chem 2011;19:926-38

24. Wei JJ, Jin L, Wan K, Zhou CH. Bull Korean Chem Soc 2011;32:229-38.

25. Sharma S, Saquib M, Verma S, Mishra NN, Shukla PK, Srivastava R, et al. Eur J Med Chem 2014;83:474-89.

26. Isloor AM, Kalluraya B, Shetty P. Eur J Med Chem 2009;44:3784-7.

27. Avendaño C, Menéndez JC. Medicinal chemistry of anticancer drugs. Amsterdam, Oxford: Elsevier; 2008.

28. Liu J, Sang C, Xu X, Zhang L, Yang X, Hui L, et al. Eur J Med Chem 2013;64:621-8. 29. Lichtenthaler FW, Peters S. C R Chim 2004;7:65-90. 
30. Queneau Y, Jarosz S, Lewandowski B, Fitremann J. Adv Carbohydr Chem Biochem 2008;61:217-92.

31. Petrova KT, Correia-da-Silva P, Crucho CIC, Barros MT. Curr Org Chem 2014; 18:1788-802.

32. Raposo CD, Petrova KT, Barros MT. Synth Commun 2014:44:3027-36.

33. Petrova KT, Potewar TM, Ascenso OS, Barros MT. Carbohydr Polym 2014;110:3846.

34. Barros MT, Petrova KT, Singh RP. Int J Mol Sci 2010;11:1792-807.

35. Barros MT, Petrova KT. Eur Polym J 2009;45:295-301.

36. Crucho CIC, Barros MT. J Mater Chem B 2014;2:3946-55.

37. Lewandowski B, Jarosz S. Org Lett 2010;12:2532-5.

38. Lewandowski B, Jarosz S. Synth Commun 2011;41:2161-8.

39. Potewar TM, Petrova KT, Barros MT. Carbohydr Res 2013;379:60-7.

40. Kumara-Swamy KC, Bhuvan-Kumar NN, Balaraman E, Pavan-Kumar KVP. Chem Rev 2009;109:2551-651.
41. Liu J, Head E, Kuratsune H, Cotman CW, Ames BN. Ann N Y Acad Sci 2004;1033: 117-31.

42. Soković MD, Vukojević J, Marin PD, Brkić DD, Vajs V, Griensven LJLDV. Molecules 2009; 14:238-49.

43. Ezabadi IR, Camoutsis C, Zoumpoulakis P, Geronikaki A, Soković M, Glamočilija J, et al. Bioorg Med Chem 2008;16:1150-6.

44. Clinical and Laboratory Standards Institute. Methods for dilution antimicrobial susceptibility tests for bacteria that grow aerobically. Approved standard, 8th ed. CLSI publication M07-A8. Clinical and Laboratory Standards Institute Wayne, PA; 2009.

45. Tsukatani T, Suenaga H, Shiga M, Noguchi K, Ishiyama M, Ezoe T, et al. J Microbiol Methods 2012;90:160-6.

46. Espinel-Ingroff A. J Clin Microbiol 2001;39:1360-7.

47. Abreu RMV, Ferreira ICFR, Calhelha RC, Lima RT, Vasconcelos MH, Adega F, et al. Eur J Med Chem 2011;46:5800-6. 